\title{
Markers for Diagnosis and Progression in Bladder Cancer ${ }^{*}$
}

\author{
Josep M. Gaya, Juan Palou", Oscar Rodríguez, Humberto Villavicencio \\ Department of Urology, Fundació Puigvert, Universitat Autònoma de Barcelona, Barcelona, Spain. \\ Email: \#jpalou@fundacio-puigvert.es
}

Received June $14^{\text {th }}, 2012$; revised July $18^{\text {th }}, 2012$; accepted July $30^{\text {th }}, 2012$

\begin{abstract}
Bladder cancer is a common disease that is often detected late and has a high rate of recurrence and progression. The current standard of care for the primary detection and follow-up of NMIBC consists of urethro-cystoscopy associated with cytology. However, several clinical risk factors have been claimed to predict recurrence and progression, these factors have a predictive value on a population basis, but no parameter has been found that reliably predicts how an individual patient's tumor will behave. In the last years many markers have been described in order to decrease the number of cystoscopies and try to provide individualized risk-stratified decision-making. We have focused our review in tumor markers for primary diagnosis, surveillance of non-muscle-invasive bladder cancer, and predicting progression to muscle-invasive disease. After our review, we can conclude that to the date no non-invasive biomarker has proven to be sensitive and specific enough to replace cystoscopy, neither in the diagnosis nor in the follow-up. On the other hand, promising results have been reported of potential biomarkers for predicting recurrence, early progression and poor response to BCG, new studies should be promoted to validate these results and make possible to incorporate markers as a new tool in clinical guidelines.
\end{abstract}

Keywords: Bladder Cancer; Non-Muscle Invasive; Recurrence; Progression; Marker; Urinary Marker; Biomarker

\section{Introduction}

Approximately 380,000 cases of bladder cancer (BC) occur around the world each year [1]. In the United States and Europe, BC is the fourth most common cancer in men [2]. This tumor affects three times as many men as women. Women, however, often have more advanced tumors than men at the time of diagnosis. While, majority of the deaths from $\mathrm{BC}$ occur in patients with muscle-invasive disease (MID), most BC (75\% - 85\%) are non-muscle invasive (NMI) at first diagnosis [3]. Generally, the prognosis for patients with NMI bladder cancer is good, although ( $30 \%-80 \%)$ will recur, and $(1 \%-45 \%)$ will progress to MID within 5 years depending on grade, stage, and associated clinical risk factors [4]. The conservative management with transurethral resection followed by intravesical treatments with chemotherapy or/and immunotherapy has demonstrated a high efficacy in patients with NMI disease decreasing the risk of recurrence and progression. It is mandatory, in patients treated conservatively, a strict, frequent, and cost follow-up [5]. Unfortunately, a significant proportion of patients of this group will not respond and will progress

\footnotetext{
${ }^{*}$ This research received no specific grant from any funding agency in the public, commercial, or not-for-profit sectors.

${ }^{\#}$ Corresponding author.
}

to invasive tumors $(\geq \mathrm{T} 2)$ despite appropriate therapy. Several studies have reported radical cystectomy is only curative in $50 \%$ of patients who progress from NMID to MID compared to $90 \%$ when this is performed in patients with superficial tumors [6]. On the other hand, this later group of patients will suffer up to $50 \%$ of overtreatment [7].

The current standard of care for the primary detection and follow-up of NMIBC consists of urethro-cystoscopy (UCS) as the gold standard and cytology as an adjunct $[3,8]$. Several clinical risk factors as: size, multifocallity, localization, associated carcinoma in situ, and recently prostatic urethra involvement and female gender $[9,10]$ have been claimed to predict recurrence and progression. But these factors have a predictive value on a population basis, but no parameter has been found that reliably predicts how an individual patient's tumor will behave.

In the last years, many markers have been described in order to improve patients' comfort and help clinicians in the primary diagnosis of this common cancer, follow-up of NMIBC, and try to provide individualized risk-stratified decision-making.

The aim of this review is to provide a summary of the current evidence of the evaluation of biomarkers for diagnosis, follow-up of NMIBC, and predicting progression to MID. The MEDLINE/PubMed databases were 
used to search for the most relevant articles both original and reviews, using different combinations of the following keywords and search terms: bladder cancer, nonmuscle-invasive bladder cancer, diagnosis, follow-up, progression, markers, urinary markers, and biomarkers.

\section{Urinary Biomarkers for Primary Detection and Surveillance of Non-Muscle-Invasive Bladder Cancer}

Urinary biomarkers may help decrease the number of cystoscopies in primary detection and surveillance, improving patients' quality-of-life and reducing the cost of follow-up. Most of the patients diagnosed of NMIBC because the risk of recurrence and progression will need a life-long follow-up by cystoscopy. Urine cytology is widely and frequently utilized but suffers from a poor sensitivity $(30 \%-35 \%)$. All new urinary biomarkers have shown a better sensitivity but lower specificity compared to cytology. The main question, still not answered, is whether sensitivity and specificity showed by these markers is enough to use them routinely and in all type of tumors, in order to avoid invasive explorations, such as cystoscopy.

Many studies do not differentiate between primary and recurrent tumors, nor is the performance of the studied marker assessed separately in NMID and MID or high versus low grade tumors. Apart from that, the vast majority of these studies are case-control trials comparing artificially composed study cohorts in which the prevalence of the disease frequently exceeds $50 \%$ [11]. Therefore, some of the findings are not reproducible when we want to translate them into routine clinical use because the efficacy of a marker is well known that depends on the studied population [12].

The most commonly studied urine markers and approved by the US Food and Drug Administration (FDA) and Health Canada (BTA, NMP-22, FISH UroVysion,

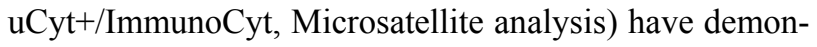
strated a high sensitivity $(58 \%-75 \%)$ and low specificity $(73 \%-86 \%)$ compared to $94 \%$ of urine cytology [13]. However, as occurs with the sensitivity of the cytology, Van Rhijn et al. [14] demonstrated in the subanalysis performed per pathological grade, that this high sensitivity decrease in the detection of low stage and grade tumors, which are the main group at primary diagnosis and recurrence. The BTA test and NMP-22 test have a very limited role because of their high false-positive rate $[15,16]$. But with careful selection of patients, the specificity of NMP-22 can be improved, and can be used during follow-up to delay cystoscopy control $[17,18]$. UroVysion can repleace cytology for high-grade tumors when experience with urinary cytology is lacking or when its result is inconclusive, but adds little to the surveillance of low-grade tumors and might be useful to predict response to intravesical therapy $[19,20]$. Immunocyt has showed the highest sensitivity for low-grade (LG) tumors and is more rarely affected by other urological disorders. However, with a $55 \%-60 \%$ detection rate for LG tumors remains inadequate to replace cystoscopy [21,22]. Microsatellite analysis can predict recurrence of LG tumors up to $80 \%$, but it still lacks sensitivity [23,24].

Nowadays, many of the tests are performed routinely in addition to urinary cytology and no single test is used widely by urologist in the follow-up of patients with BC. The trend is to associate one or more urine markers with urine cytology in order to improve sensitivity without decreasing specificity. Li, H. X. et al. [25] recently demonstrated that using Immunocyt and Cytokeratin $20 \mathrm{im}-$ munochemistry as adjunct marker for cytology were able to improve the sensitivity of cytology in detecting UC, specially for low grade, without a significant decrease in specificity. Other non-FDA approved, urine-based test are being investigated as potential urothelial carcinoma biomarkers include: citokeratins-8-19-20, microsatellite mutations, telomerase, DNA methylation and histone acetylation [26-29]. But to the date no non-invasive biomarker has proven to be sensitive and specific enough to replace cistoscopy, neither in the diagnosis nor in the follow-up. Additional studies are needed to determinate the clinical scenarios were urinary markers can be useful. The future markers need to be better, easier to perform, cheaper, and faster for the interpretation of the results. The test should have a high positive predictive value to avoid unnecessary invasive explorations, and high negative predictive value to avoid the risk of failing to detect tumors. The main problem is that these parameters vary between populations with different incidences. Therefore, cannot be used for comparing methods and tests.

\section{Biomarkers for Predecting Recurrence and Progression to Muscle-Invasive Disease in NMIBC}

Bladder cancer is a genetic disorder driven by progresssive accumulation of multiple genetic changes. The specific alterations in gene expression that occur as a result of cross-talk between various cellular pathways determinate the biologic behavior of the tumor. Thus different molecular profiles have been associated with recurrence, early progression and the response to a specific therapy. Biological measures may help to elicit the individual patient's risk of future outcome. Hence, they may be useful to identify those patients with NMIBC who could benefit from immediate radical cystectomy and do not waste a critical time with conservative management or ones who should have a more strict follow-up.

Because of all of this, in the last years the search for biomarkers has been called into question and has ob- 
tained notoriety in basic and transitional $\mathrm{BC}$ research. Recently several studies, with significant number of cases, have reported that different biomarkers, in diverse cellular function, should be taken in consideration as predictors for recurrence and progression to MID in patients with NMIBC. These studies include: Karyopherin$\alpha 2$, a nuclear and cytoplasmatic protein which is thought to play and important role in the nucleocytoplasmatic transport and has also been suggested to be a transporter of tumor supressors [30]; HMOX 1, one of the three isoforms of HMOX that catalyzes the degradation oh heme to biliverdin, carbon monoxide and free iron [31]. GSTM1 and GSTT1 both polymorfisms in glutathione S-transferase enzym [32,33]; C16orf74 a gene involved in inflammatory processes and strongly associated with an anti-TNF response and hypoxia [34]; and the well known HRAS, KRAS, p63, Her-2 [35,36]. Her-2 protein expression has been recently published as Ezrin protein expression and the methylation status of TSGs were able to distinguish patients responding to BCG from those who may require more aggressive therapeutic intervenetion [37-39]. The p53 mutation and p53 protein status were one of the first markers in showing promising results for predicting outcomes of patients with NMIBC, but finally they have been abandoned for clinical practice, even though many studies continue to show significant association between recurrence, progression and survival $[40,41]$.

Although several biomarkers obtained from these different studies have showed promising results, the majority of the experts in the field consider it is unlikely that any single marker will be able to improve prognostication for patients with NMIBC. Similary is happening with clinical risk factors, many of the authors advocated for a combination of multiple independent markers to predict outcome. Bryan et al. [42] published in 2005 a panel using seven independent biomarkers according to increased expression of proteins and grow factors who play critical and different roles in regulation of the cell cycle, apoptosis, DNA synthesis and repair, and angiogenesis. The same group in 2009 updated their results and confirmed that the combination of (VEFG, pRb, FGFR3, CK-20, Ki-67, EGFR and p53) was effective to predict, recurrence, progression, and poor outcome in patients with BC.

Other authors have proposed and present their good results using a group of markers involved in one pathway (apoptosis, cell cycle regulation, etc.) [43,44].

Since a role for inflammation in carcinogenesis is now well-established) some authors [45] have worked in the analysis of COX-2 expression. But it seems that even COX-2 expression is higher in high grade and MID compared to NMID, but this is not useful to predict progression in NMIBC.
Although multiple markers described, any one has been included in guidelines to help clinicians into clinical decision-making, and clinical risk factors are still the only risk calculator tool to decide conservative management versus radical cystectomy in high risk NMIBC.

\section{Conclusions-Take Home Message}

In general, the best urinary markers have higher sensitivity than urinary cytology, but specificity is usually lower. But to the date no non-invasive biomarker has proven to be sensitive and specific enough to replace cistoscopy, neither in the diagnosis nor in the follow-up. For this reason, UCS continues to be the mainstay in BC detection and follow-up. As cytology is highly specific, it is still important as and adjunct for the evaluation of patients with hematuria, in the detection of primary $\mathrm{BC}$ and also in the surveillance of patients with high grade NMIBC. However, new markers combinations associated with urinary cytology may play a role in future screening of patients with low grade NMIBC.

Many markers for predicting recurrence, early progression and poor response to $\mathrm{BCG}$ have been identified in the last years. While some authors proposed using a combination of them, others advocate for individual ones. Due to the fact that classical clinical risk factors are not specific enough for predicting NMIBC outcomes and that promising results have been reported of potential biomarkers, new studies should be promoted to validate these results and make possible to incorporate markers as a new tool in clinical guidelines. But to carry out the translation of knowledge into practice with maximal efficiency and effectiveness, it is essential to conduct these studies with appropriate designs.

\section{REFERENCES}

[1] D. M. Parkin, F. Bray, J. Gerlay, et al., "Global Cancer Statistics, 2002," CA: A Cancer Journal for Clinicians, Vol. 55, No. 2, 2005, pp. 74-108. doi:10.3322/canjclin.55.2.74

[2] M. Grasso, "Bladder Cancer: A Major Public Health Issue," European Urology Supplements, Vol. 7, No. 7, 2008, pp. 510-515. doi:10.1016/j.eursup.2008.04.001

[3] M. Babjuk, W. Oosterlinck, R. Sylvester, E. Kaasinen, A. Böhle, J. Palou-Redorta and M. Rouprêt, "European Guidelines on Non-Muscle Invasive Bladder Cancer TaT1 and CIS," European Urology, Vol. 59,No. 6, 2011, pp. 997-1008.

[4] R. J. Sylvester, A. P. Van der Meijden, W. Oosterlinck, et al., "Predicting Recurrence and Progression in Individual Patients with Stage TaT1 Bladder Cancer Using EORTC Risk Tables: A Combined Analysis of 2596 Patients from Seven EORTC Trials," European Urology, Vol. 49, No. 3, 2006, pp. 466-477. doi:10.1016/j.eururo.2005.12.031

[5] R. J. Sylvester, A. Van der Meijden, D. L. Lamm, et al., 
"Intravesical Bacillus Calmette-Guerin Reduces the Risk of Progression in Patients with Superficial Bladder Cancer: A Meta-Analysis of the Published Results of Randomized Clinical Trials," The Journal of urology, Vol. 168, No. 5, 2002, pp. 1964-1970. doi:10.1016/S0022-5347(05)64273-5

[6] J. Huguet, J. M. Gaya, S. Sabate, et al., "Radical Cystectomy in Patients with Non-Muscle Invasive Bladder Cancer Who Fail BCG Therapy," Actas Urológicas Españolas, Vol. 34, No. 1, 2010, pp. 63-70. doi:10.1016/S0210-4806(10)70011-5

[7] C. L. Amling, J. B. Thrasher, H. A. Frazier, et al., "Radical Cystectomy for Stages Ta, Tis and T1 Transitional Cell Carcinoma of the Bladder," The Journal of Urology, Vol. 151, No. 1, 1994, pp. 31-36.

[8] M. Brausi, J. A. Witjes, D. Lamm, R. Persad, J. Palou, M. Colombel, R. Buckley, M. Soloway, H. Akaza and A. Böhle, "A Review of Current Guidelines and Best Practice Recommendations For the Managment of Nonmuscle Invasive Bladder Cancer by the International Bladder Cancer Group," European Urology, Vol. 186, No. 6, 2011, pp. 2158-2167.

[9] F. Millan-Rodriguez, G. Chechile-Toniolo, J. SalvadorBayarri, et al., "Multivariate Analysis of the Prognostic Factors of Primary Superficial Bladder Cancer," The Journal of Urology, Vol. 163, No. 1, 2000, pp. 73-78. doi:10.1016/S0022-5347(05)67975-X

[10] J. Palou, J. M. Gaya, R. Sylvester, et al., "Prognostic Factors for Recurrence and Progression in T1G3 Bladder Cancer Treated with BCG: CIS in the Prostatic Urethra and Gender," European Urology Supplements, Vol. 8, No. 4, 2009, p. 285. doi:10.1016/S1569-9056(09)60653-9

[11] Y. Lotan, S. F. Shariat, B. J. Schmitz-Dräger, et al., "Considerations on Implementing Diagnostic Markers into Clinical Decision Making in Bladder Cancer," Urologic Oncology: Seminars and Original Investigations, Vol. 28, No. 4, 2010, pp. 441-448.

[12] Y. Lotan, S. F. Shariat, et al., (NMP22 Study Group), "Impact of Risk Factors on the Performance of the Nuclear matrix Proteína 22 Point-of-Care-Test for Bladder Cancer Detection," BJU International, Vol. 101, No. 11, 2008, pp. 1362-1367. doi:10.1111/j.1464-410X.2008.07473.x

[13] V. B. Lobeshwar, T. Habuchi, H. B.Grossman, et al., "Bladder Tumor Markers Consensus Panel," Urology, Vol. 66, Suppl. 6, 2005, pp. 35-63.

[14] B. Van Rhijin, H. Van der Poel, T. Van der Kwast, et al., "Citology and Urinary Markers for the Diagnosis of Bladder Cancer," European Urology Supplements, Vol. 8, No. 7, 2009, pp. 536-541. doi:10.1016/j.eursup.2009.06.008

[15] M. Babjuk, V. Soukup, M. Pesl, et al., "Urinary Cytology and Quantitative BTA and UBC Tests in Surveillance of Patients with pTapT1 Bladder Urothelial Carcinoma," Urology, Vol. 71, No. 4, 2008, pp. 718-722. doi:10.1016/j.urology.2007.12.021

[16] M. P. Raitanen, "The Role of BTA Stat Test in FollowUp of Patients with Bladder Cancer: Results from FinnBladder Studies," World Journal of Urology, Vol. 26, No.

\section{1, 2008, pp. 45-50. doi:10.1007/s00345-007-0230-3}

[17] Y. Lotan, S. F. Shariat and N. S. Grp, "Impact of Risk Factors on the Performance of the Nuclear Matrix Protein 22 Point-of-Care Test for Bladder Cancer Detection," BJU International, Vol. 101, No. 11, 2008, pp. 1362 1367. doi:10.1111/j.1464-410X.2008.07473.x

[18] C. T. Nguyen and J. S. Jones, "Defining the Role of NMP22 in Bladder Cancer Surveillance," World Journal of Urology, Vol. 26, No. 1, 2008, pp. 51-58. doi:10.1007/s00345-007-0226-Z

[19] B. J. Schlomer, R. Ho, A. Sagalowsky, et al., "Prospective Validation of the Clinical Usefulness of Reflex Fluorescence in Situ Hybridization Assay in Patients with Atypical Cytology for the Detection of Urothelial Carcinoma of the Bladder," The Journal of Urology, Vol. 183, No. 1, 2010, pp. 62-67.

[20] J. Bergman, R. C. Reznichek and J. Rajfer, "Surveillance of Patients with Bladder Carcinoma Using Fluorescent in-Situ Hybridization on Bladder Washings," BJU International, Vol. 101, No. 1, 2008, pp. 26-29.

[21] G. Mowatt, S. Zhu, M. Kilonzo, et al., "Systematic Review of the Clinical Effectiveness and Cost-Effectiveness of Photodynamic Diagnosis and Urine Biomarkers (FISH, ImmunoCyt, NMP22) and Cytology for the Detection and Follow-Up of Bladder Cancer," Health Technol Assess, Vol. 14, No. 4, 2010, pp. 1-331.

[22] B. J. Schmitz-Drager, B. Beiche, L. A. Tirsar, et al., "Immunocytology in the Assessment of Patients with Asymptomatic Microhaematuria," European Urology, Vol. 51, No. 6, 2007, pp. 1582-1588. doi:10.1016/j.eururo.2006.10.046

[23] M. N. M. van der Aa, E. C. Zwarthoff, E. W. Steyerberg, et al., "Microsatellite Analysis of Voided-Urine Samples for Surveillance of Low-Grade Non-Muscle-Invasive Urothelial Carcinoma: Feasibility and Clinical Utility in a Prospective Multicenter Study (Cost-Effectiveness of Follow-Up of Urinary Bladder Cancer Trial [CEFUB])," European Urology, Vol. 55, No. 3, 2009, pp. 659-667. doi:10.1016/j.eururo.2008.05.001

[24] E. W. de Bekker-Grob, M. N. van der Aa, E. C. Zwarthoff, et al., "Non-Muscle-Invasive Bladder Cancer Surveillance for Which Cystoscopy is Partly Replaced by Microsatellite Analysis of Urine: A Cost-Effective Alternative?" BJU International, Vol. 104, No. 1, 2009, pp. 41-47. doi:10.1111/j.1464-410X.2008.08323.x

[25] H. X. Li, M. Li, L. C. Li, et al., "Immunocyt and Cytokeratin 20 Immunochemistry as Adjunt Marker for Urine Cytologic Detection of Baldder Cancer: A Prospective Study," Analytical and Quantitative Cytology and Histology, Vol. 32, No. 1, 2010, pp. 45-52.

[26] F. Barbisan, A. Santinelli, R. Mazzucchelli, et al., "Strong Immunohistochemichal Expression of Fibroblast Growth Factor Receptor 3 Superficial Staining Pattern of Cytokeratin 20, Low Proliferative Activity Define Those Papillary Neoplasms of Low Malignant Potencial That Do Not Recur," Cancer, Vol. 112, No. 3, 2008, pp. 636644.

[27] G. Legrand, H. Soliman, F. Dubosq, et al., "Prevalence 
and Spectrum of Microsatellite Alterations in Nonmuscle Invasive Bladder Cancers," American Journal of Cancer Research, Vol. 1, No. 5, 2011, pp. 595-603.

[28] R. T. Bryan, S. A. Hussain, N. D. James, et al., "Molecular Pathway in Bladder Cancer. Part 1," BJU International, Vol. 95, No. 4, 2005, pp. 485-490. doi:10.1111/j.1464-410X.2005.05325.x

[29] R. T. Bryan, S. A. Hussain, N. D. James, et al., "Molecular Pathway in Bladder Cancer. Part 2," BJU International, Vol. 95, No. 4, 2005, pp. 491-496 doi:10.1111/j.1464-410X.2005.05326.x

[30] J. B. Jensen, P. P.Munksgard, C. M.Sorensen, et al., "High Expression of Karyopherin-x2 Defines Poor Prognosis in Non-Muscle-Invasive Bladder Cancer and in Patients with Invasive Bladder Cancer Undergoing Radical Cystectomy," European Urology, Vol. 59, No. 5, 2011, pp. 841-848. doi:10.1016/j.eururo.2011.01.048

[31] M. Yim, Y. Ha, I. Kim, et al., "HMOX1 is an Important Prognostic Indicator of Non-Muscle Invasive Bladder Cancer Recurrence and Progresión," The Journal of Urology, Vol. 185, No. 2, 2011,pp. 701-705. doi:10.1016/j.juro.2010.09.081

[32] Y. S. Ha, C. Yan, P. Jeong, et al., "GSTM1 Tissue Genotype as a Recurrence Predictor in Non-Muscle Invasive Bladder Cancer," Journal of Korean Medical Science, Vol. 26, No. 2, 2011, pp. 231-236. doi:10.3346/jkms.2011.26.2.231

[33] Y. S. Ha, C. Yan, M. Lym, et al., "GSTT1 as a Prognosticator for Recurrence and Progresión in Patients with Non-Muscle-Invasive Bladder Cancer," Disease Markers, Vol. 29, No. 2, 2010, pp. 81-87.

[34] W. T. Kim, S. J. Yon, C. Park, et al., "Identification of c16orf74 as a Marker of Progresión in Primary NonMuscle Invasive Bladder Cancer," PLoS One, Vol. 5, No. 12, 2010, p. e15260.

[35] L. C. Kompier, I. Lurkin, M. N. M. van der Aa, et al., "FGFR3, HRAS, KRAS,NRAS and PIK3CA Mutations in Bladder Cancer and Their Potential as Biomarkers for Surveillance and Therapy," PLoS One, Vol. 5, No. 11, 2010, p. e13821. doi:10.1371/journal.pone.0013821

[36] O. Karni-Schmidt, M. Castillo-Martin, T. H. Shen, et al., "Distinct Expresión Profiles of p63 Variants Turing Urothelial Development and Bladder Cancer Progresión," The American Journal of Pathology, Vol. 178, No. 3, 2011, pp. 1350-1360. doi:10.1016/j.ajpath.2010.11.061
[37] A. Janane, F. Hajji, T. O. Ismail, et al., "Evaluation of HER2 Protein Overexpression in Non-Muscle-Invasive Bladder Cancer with Emphasis on Tumor Grade and Recurrence," Actas Urológicas Españolas, Vol. 35, No. 4, 2011, pp. 189-194. doi:10.1016/S2173-5786(11)70048-8

[38] J. Palou, F. Algaba, I. Vera, M. Sánchez-Carbayo, et al., "Protein Expression Patterns of Ezrin Are Predictors of Progresión in T1G3 Bladder Tumors Treated with NonMaintenance Bacillus Calmette-Guérin," European Urology, Vol. 56, No. 5, 2009, pp. 829-836. doi:10.1016/j.eururo.2008.09.062

[39] M. Agundez, L. Grau, J. Palou, et al., "Evaluation of the Methylation Status of Tumor Supressor Genes for Predicting Bacillus Calmette-Guérin Response in Patients with T1G3 High Risk Bladder Tumours," European Urology, Vol. 60, No. 1, 2011, pp. 131-140. doi:10.1016/j.eururo.2011.04.020

[40] B. George, R. H. Datar, L. Wu, et al., "p53 Gene and Proteína Status: The Role of p53 Alterations in Predicting Outcome in Patients with Bladder Cancer," Journal of Clinical Oncology, Vol. 25, No. 34, 2007, pp. 5352-5358. doi:10.1200/JCO.2006.10.4125

[41] A. S. Salinas-Sánchez, J. G. Lorenzo-Romero, J. M. Giménez-Bachs, et al., "Implications of p53 Gene Mutations on Patient Survival in Transicional Cell Carcinoma of the Bladder: A Long Term Study," Urologic Oncology: Seminars and Original Investigations, Vol. 26, No. 6, 2008, pp. 620-626. doi:10.1016/j.urolonc.2007.07.011

[42] R. T. Bryan, P. Zeegers, N. D. James, et al., "Biomarkers in Bladder Cancer," BJU International, Vol. 57, No. 1, 2009, pp. 1-13. doi:10.1111/j.1464-410X.2009.08880.x

[43] P. Mhawech-Fanceglia, G. Fischer, V. Alvarez Jr., et al., "Predicting Outcome in Minimally Invasive (T1a and T1b) Urothelial Bladder Carcinoma Using a Panel of Biomarkers: A High Throughput Tissue Microarray Analisis," BJU International, Vol. 100, No. 5, 2007, pp. 1182-1187.

[44] A. W. Hitchings, M. Kumar, S. Jordan, et al., "Prediction of Progression in pTa and pT1 Bladder Carcinomas with p53,16 and pRb," British Journal of Cancer, Vol. 91, No. 3, 2004, pp. 552-557. doi:10.1038/sj.bjc.6601954

[45] K. Diamantopoulou, A. Lazaris, E. Mylona, et al., "Cycloxigenase-2 Protein Expression in Relation to Apoptotic Potential and Its Prognostic Significance in Bladder Urothelial Carcinoma," Anticancer Research, Vol. 25, No. 6, 2005, pp. 4543-4549. 